\title{
El uso de terapia hormonal de reemplazo aumenta el riesgo de enfermedad tromboembólica venosa
}

Postmenopausal hormone therapy increases risk for venous thromboembolic disease. The Heart and Estrogen/progestin Replacement Study. Ann Intern Med. 2000 May 2;132(9):689-96

\section{Objetivo}

Determinar el efecto del tratamiento con estrógenos y progestágenos en el riesgo de desarrollar eventos tromboembólicos venosos en mujeres postmenopáusicas.

\section{Diseño}

Estudio prospectivo, randomizado, doble ciego, controlado con placebo

\section{Lugar}

20 centros clínicos de Estados Unidos.

\section{Participantes}

2763 mujeres postmenopáusicas menores de 80 años con enfermedad coronaria, sin historia previa de enfermedad tromboembólica venosa, que no estuvieran histerectomizadas.

\section{Intervención}

Se administró $0.625 \mathrm{mg}$ de estrogenos conjugados y $2.5 \mathrm{mg}$ de medroxiprogesterona en una tableta $(n=1380)$ o placebo de aspecto similar $(n=1383)$.

\section{Medición de los objetivos}

Documentación de trombosis venosa profunda o tromboembolismo pulmonar.

\section{Resultados}

La duración media del seguimiento fue de 4.1 años. La incidencia de eventos tromboembólicos venosos fue mayor en el grupo que recibió tratamiento hormonal siendo el RR 2.7 (IC95\%:1.4-5.0) ( $\mathrm{p}: 0.003$ ), con un exceso de riesgo de 3.9 por 1000 mujeres-año (IC95\%: 1.4 6.4). En el análisis multivariado, el riesgo de tromboembolismo venoso estuvo incrementado en las mujeres con fracturas de miembros inferiores (RR: 18.1[IC: 5.4 - 60.4]), cáncer (RR: 3.9 [IC:1.6 9.4]); 90 días posteriores a procedimientos quirúrgicos (RR: 4.9 [2.4 - 9.8]) u hospitalización no quirúrgica (RR: 5.7 [IC:3.0 - 10.8]). El riesgo disminuyó con el uso de aspirina (RR: 0.5 [IC:0.2 - 0.8]) o estatinas (RR: 0.5 [IC: $0.2-0.9]$ ).

\section{Conclusiones}

El uso de tratamiento de reemplazo hormonal con estrógenos y progestágenos incrementa el riesgo de tromboembolismo venoso en mujeres con enfermedad coronaria.

\section{COMENTARIO}

El incremento del riesgo trombótico venoso con el uso de terapia de reemplazo hormonal (TRH) ha sido ampliamente documentado en varios trabajos 1,2. En este estudio se analiza el riesgo de desarroLlar enfermedad tromboembólica venosa (ETV) con el uso de TRH en mujeres postmenopausicas con enfermedad coronaria, hecho que resulta importante para el análisis de la toma de decisión. Se evalúa una población seleccionada, confirmándose el aumento en el riesgo a desarrollar ETV con la terapia de reemplazo hormonal, siendo el RR 2.7 (IC95\%:1.4-5.0), con un exceso de 3.9 eventos por cada 1000 mujeres año, necesitándose tratar 256 mujeres durante 1 año para desarrollar un evento trombótico adicional. Resulta fundamental conocer si este efecto de la terapia de reemplazo tiene igual peso en forma independiente o cobra mayor importancia al. potenciar la presencia de otros factores de riesgo. En la incidencia de trombosis idiopática, el RR fue de $3.1(0.8-11.3)$, p=0.09. Cuando los eventos fueron asociados a factores predisponentes, el RR fue de 2.5 (1.2-5.3), $p=0.01$. El riesgo de tromboembolismo venoso aumentó en presencia de factores predisponentes como la fractura de miembros inferiores y de cadera, cáncer, los 90 días posteriores a la internación quirúrgica y no quirúrgica. También en este trabajo se observa una disminución del riesgo para desarrollar
ETV con la aspirina (RH: 0.5 [IC:0.2 - 0.8]). La eficacia del uso de aspirina para disminuir el riesgo a desarrollar ETV es menor que la demostrada por la heparina ${ }^{3}$, sin embargo recientemente el estudio PEP4 que randomizó 13.356 pacientes a recibir aspirina o placebo, demostró su utilidad en la profilaxis de la ETV, aún excluyendo del análisis su actividad sobre los eventos cardiovasculares que potencialmente pueden generar ETV, como el accidente cerebrovascular (ACV) y el infarto de miocardio (IAM). Si bien en el trabajo que estamos analizando, hubo incremento de ETV asociada a IAM (RR: 3.6 [1.5-8.7]) y a ACV(RR: 4.2 [1.6-10.8], su significancia se pierde en el análisis multivariado, donde se demuestra una reducción de la ETV con el uso de aspirina en forma independiente. Con respecto a las estatinas se encontró una disminución del riesgo, RR: 0.5 [IC: $0.2-0.9$ ], que no se ha documentado previamente, por lo que se necesitan mas estudios para confirmar este efecto. El incremento del riesgo a desarrollar ETV debe ser considerado al momento de evaluar la indicación de la terapia de reemplazo, siendo necesaria la exclusión de los casos con presencia de factores predisponentes permanentes y la implementación adicional de medidas profilácticas ante la aparición de factores predisponentes transitorios de ETV.

\section{Dr. Jorge Arbelbide}

Sección Hematología, servicio de Clínica Médica. Hospital Italiano de Buenos Aires.

\section{Referencias}

1. Castellsague J, Perez Gutthann S, Garcia Rodriguez LA. Recent epidemiological studies of the association between hormone replacement therapy and venous thromboembolism. A review. Drug Saf. 1998 Feb;18(2):117-23

2. Douketis JD, Ginsberg JS, Holbrook A, Crowther M, Duku EK, Burrows RF.A reevaluation of the risk for venous thromboembolism with the use of oral contraceptives and hormone replacement therapy. Arch Intern Med. 1997 Jul 28;157(14):1522-30.

3. Clagett GP, Anderson FA Jr, Geerts W, et al. Prevention of venous thromboembolism. Chest 1998; 114:531S-60S.

4. Prevention of pulmonary embolism and deep vein thrombosis with Low dose aspirin: Pulmonary Embolism Prevention (PEP) trial. Lancet. 2000 Apr 15;355(9212):1295-302. 